\title{
Progênies superiores de soja resistentes ao tipo 3 do nematóide de cisto da soja
}

\author{
Roberto Carlos de Oliveira(1), Antonio Orlando Di Mauro(1), Sandra Helena Unêda-Trevisoli(1), \\ Jaime Maia dos Santos ${ }^{(1)}$, João Ademir de Oliveira ${ }^{(1)}$, Dilermando Perecin ${ }^{(1)}$ e Neylson Eustáquio Arantes ${ }^{(2)}$
}

\begin{abstract}
(1)Universidade Estadual Paulista, Fac. de Ciências Agrárias e Veterinárias, Via de Acesso Prof. Paulo Donato Castellane, s/no CEP 14884-900 Jaboticabal, SP. E-mail: rcoliveira@netsite.com.br, orlando@fcav.unesp.com.br, shutrevi@fcav.unesp.com.br, jmsantos@fcav.unesp.br, jaoliv@fcav.unesp.br, perecin@fcav.unesp.br (2)Embrapa Soja, Rod. Carlos João Strass, Distrito de Warta, CEP 86001-970 Londrina, PR. E-mail: neylson@epamiguberaba.com.br
\end{abstract}

\begin{abstract}
Resumo - Os objetivos deste trabalho foram selecionar progênies superiores de soja e avaliá-las, em casa de vegetação, quanto à resistência ao nematóide tipo 3 de cisto da soja (Heterodera glycines). Foram avaliadas 222 progênies segregantes de soja, em ensaios conduzidos em campo, nos anos 1999/2000, 2000/2001 e 2001/2002, sob delineamento de blocos aumentados de Federer, e no ano 2002/2003 em blocos ao acaso, com duas repetições, tendo sido avaliados dez atributos agronômicos. No ano de 2003 foi conduzido em casa de vegetação um ensaio com 11 progênies superiores, para a avaliação de resistência ao nematóide de cisto, adotando-se delineamento inteiramente casualizado, com cinco repetições. Com relação aos atributos agronômicos, as progênies JAB 99-17-4-9-1 e JAB 99-40-12-1-2 destacaram-se das demais por possuir médias adequadas para a maioria dos atributos. Por sua vez, na avaliação de resistência em casa de vegetação, seis progênies revelaram-se resistentes ao nematóide de cisto da soja tipo 3.
\end{abstract}

Termos para indexação: Glycine max, Heterodera glycines, melhoramento.

\section{Soybean superior progenies with resistance to type 3 soybean cyst nematode}

\begin{abstract}
The aim of this work was to select in greenhouse soybean superior progenies resistant to type 3 soybean cyst nematode (Heterodera glycines). Ten agronomic traits were evaluated in 222 segregate progenies in field. In crop seasons of 1999/2000, 2000/2001 and 2001/2002, experiments were carried out in augmented blocks of Federer, and in 2002/2003 in a randomized blocks design, with two replications. In 2003 another experiment was carried out in greenhouse, under completely randomized design with five replications and with 11 superior progenies, in order to evaluate resistance against cyst nematode. Progenies JAB 99-17-4-9-1 and JAB 99-40-12-1-2 scored the best results for most of the agronomic traits evaluated. Under greenhouse conditions six progenies were resistant to type 3 soybean cyst nematode.
\end{abstract}

Index terms: Glycine max, Heterodera glycines, plant breeding.

\section{Introdução}

A soja é uma das principais oleaginosas produzidas no mundo e matéria prima empregada na elaboração de diversos produtos, desde óleo até papel. Essa leguminosa, em função de seu valor econômico e de sua potencialidade de cultivo, em diversas condições, tem apresentado constante desenvolvimento tecnológico, o que permitiu, entre outras coisas, aumento significativo de sua produtividade e de expansão de fronteiras agrícolas. Atualmente, o Brasil é o segundo maior produtor mundial de soja, com 50,3 milhões de toneladas no ano agrícola 2002/2003, e uma produtividade média de aproximadamente $2.680 \mathrm{~kg} \mathrm{ha}^{-1}$ (Conab, 2004).

Como conseqüência de sua exposição a diversos ambientes, tem ocorrido aumento na incidência de doenças, tanto em número como em gravidade. Entre as mais graves, está a ocasionada pelo nematóide de cisto da soja (NCS), Heterodera glycines Ichinohe, 1952, considerada uma das mais sérias ameaças às lavouras de soja do país. Na safra 1999/2000, as doenças da soja foram responsáveis por prejuízos estimados em US \$ 1,39 bilhão. Desse montante, o nematóide de cisto da soja (NCS) foi causador de um dano de US\$ 133,2 milhões (Yorinori, 2000). 
O melhoramento genético de plantas tem sido uma ferramenta importante, não apenas para o desenvolvimento de cultivares com bom desempenho agronômico e obtenção de ganhos genéticos, como também na eliminação de fatores restritivos à produtividade, principalmente pela incorporação de resistência a doenças (Arantes et al., 1999). O desenvolvimento de cultivares resistentes está entre as alternativas mais eficazes e econômicas para solucionar esses problemas, além de reduzir o impacto ambiental, pela minimização na utilização de insumos.

Nos últimos anos foram lançadas várias cultivares resistentes ao nematóide de cisto da soja. Em 1997 foi lançada a BRSMG Renascença, primeira cultivar brasileira resistente ao NCS (Arantes et al., 1999). No ano de 1998 a Embrapa Soja e seus parceiros lançaram as cultivares BRSMG Liderança e BRSMT Pintado. Ainda em 1998, a Monsoy Sementes lançou as cultivares M-SOY 8001 e M-SOY 8401 (Embrapa, 1998). Recentemente foram lançadas outras cultivares resistentes: BRSMG Preciosa, BRSMG Robusta, resistentes ao tipo 3; BRSMT Tucunaré, BRSMT Caxara, BRSMT Matrinchã, BRSMT Piraíba, resistentes aos tipos 1 e 3; BRSGO Chapadões, resistente aos tipos 1, 3, 4 e 14 e BRSGO Ipameri, resistente aos tipos 3 e 14, dentre outras (Dias, 2003).

Este trabalho teve como objetivos selecionar progênies de soja portadoras de atributos agronômicos desejáveis e avaliá-las, em casa de vegetação, quanto à resistência ao nematóide de cisto da soja (NCS).

\section{Material e Métodos}

Os ensaios de seleção de progênies, quanto aos atributos agronômicos, e a avaliação de resistência ao nematóide de cisto, foram realizados no campus da Faculdade de Ciências Agrárias e Veterinárias de Jaboticabal, Unesp, onde o solo é do tipo Latossolo Vermelho eutrófico, de textura argilosa, e o relevo é suavemente ondulado.

As progênies foram avaliadas nos anos agrícolas de 1999/2000, 2000/2001 e 2001/2002, em ensaios conduzidos no delineamento de blocos aumentados de Federer. Em virtude do número limitado de sementes por progênie, nas gerações iniciais, foi adotado o referido delineamento, que se caracteriza por não apresentar repetições, e o resíduo foi estimado a partir das testemunhas, as quais eram comuns. As parcelas foram constituídas por uma fileira de $5 \mathrm{~m}$ de comprimento e espaçadas em $0,5 \mathrm{~m}$.
No ano agrícola 2002/2003, o delineamento estatístico utilizado foi o de blocos ao acaso, com duas repetições; cada parcela foi composta por duas fileiras de $5 \mathrm{~m}$ de comprimento, espaçadas em 0,5 m, cuja área útil compreendeu somente os $4 \mathrm{~m}^{2}$ centrais. Foram utilizadas, como testemunhas, as cultivares Liderança, FT-Cristalina e Renascença.

A semeadura foi realizada no mês de novembro, em sulcos, com o solo previamente preparado de maneira convencional, com uma aração profunda e duas gradagens. A adubação foi efetuada diretamente nos sulcos de plantio, previamente abertos com 6-8 cm de profundidade, empregando-se $300 \mathrm{~kg} \mathrm{ha}^{-1}$ da fórmula NPK 4-20-20. Antes da inoculação de Bradyrhyzobium japonicum (Kirchner 1896) Jordan 1982, as sementes foram tratadas com fungicida Thiram, na dosagem $75 \mathrm{~g}$ por $100 \mathrm{~kg}$.

Encontra-se na Tabela 1 a relação das 222 progênies, oriundas de 18 cruzamentos, pertencentes ao programa de melhoramento de soja, conduzido no Departamento de Produção Vegetal da Fcav/Unesp, Jaboticabal, SP.

As observações foram realizadas em seis plantas da área útil, em todos os ensaios, tendo sido avaliadas as seguintes variáveis: número de dias para florescimento (NDF), número de vagens (NV), peso total por planta (PT), peso de cem sementes (PCS), número de dias para maturidade (NDM), altura de planta na maturidade (APM), altura da inserção da primeira vagem (AIV), acamamento (Ac), valor agronômico (VA) e número de nós (NN).

Às variáveis acamamento e valor agronômico, em que as notas variam de 1 a 5 , foram atribuídas notas intermediárias, entre os valores inteiros de nota: 1,$5 ; 2,5$; 3,5 e 4,5. Antes de serem efetuadas as análises estatísticas, os dados referentes a essas duas variáveis foram transformados para $\sqrt{x}$.

Uma análise de variância conjunta, que envolveu todos os ensaios instalados, nos quatro anos agrícolas, foi efetuada, utilizando-se somente os tratamentos comuns a todos os anos, ou seja, onze progênies e três testemunhas. O modelo matemático adotado foi o seguinte: $\mathrm{Y}_{\mathrm{ijklm}}=\mu+\mathrm{c}_{\mathrm{i}}+\mathrm{g} / \mathrm{c}_{\mathrm{j}(\mathrm{i})}+\mathrm{a}_{\mathrm{k}}+\mathrm{p}_{\mathrm{l}}+\mathrm{t}_{\mathrm{m}}+\mathrm{c}+(\mathrm{pa})_{\mathrm{lk}}+\mathrm{ca}+$ $e_{i j k l m}$, em que $Y_{i j k l m}$ é a observação referente ao conjunto i, na geração j, no ano k, na progênie l e na testemunha m; $\mu$ é a média geral do caráter; $c_{\mathrm{i}}$ é o efeito fixo do conjunto i, sendo $i=1, \ldots, 2 ; g / c_{j(i)}$ é o efeito fixo da geração j dentro do conjunto $i$, sendo $j=1, \ldots, 4 ; a_{k}$ é o 
efeito aleatório do ano $\mathrm{k}$, sendo $\mathrm{k}=1, \ldots, 4$; $\mathrm{p}_{\mathrm{l}}$ é o efeito fixo da progênie $l$, sendo $\mathrm{l}=1, \ldots, 11 ; \mathrm{t}_{\mathrm{m}}$ é o efeito fixo da testemunha $\mathrm{m}$, com $\mathrm{m}=1, \ldots, 3$; $\mathrm{c}$ é o efeito fixo do contraste da média das progênies $\mathrm{x}$ a média das testemu-

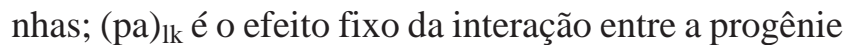
l e o ano k; ca é o efeito fixo da interação entre o ano e o contraste da média das progênies $\mathrm{x}$ a média das testemunhas; $\mathrm{e}_{\mathrm{ijklm}}$ é o erro experimental considerando-se

Tabela 1. Relação das progênies, número de progênies avaliadas, gerações, cruzamentos e reação dos parentais, quanto ao NCS (tipo 3).

\begin{tabular}{|c|c|c|c|c|}
\hline Ano agrícola & Progênie & $\mathrm{N}^{\mathrm{o}}$ de progênies avaliadas & Geração & Cruzamento/Reação ao $\mathrm{NCS}^{(1)}$ \\
\hline \multirow[t]{18}{*}{$1999 / 2000$} & JAB 99-10 & 1 & $\mathrm{~F}_{3}$ & FT-Cristalina (S) x BR 92-15440 (R) \\
\hline & JAB 99-11 & 1 & $\mathrm{~F}_{3}$ & BR 91-10569 (R) x FT-Cristalina (S) \\
\hline & JAB 99-12 & 1 & $\mathrm{~F}_{3}$ & BR 92-15440 (R) x FT-Cristalina (S) \\
\hline & JAB 99-13 & 1 & $\mathrm{~F}_{3}$ & Delsoy (R) x FT-Cristalina (S) \\
\hline & JAB 99-14 & 1 & $\mathrm{~F}_{3}$ & BR 90-4617 (R) x FT-Cristalina (S) \\
\hline & JAB 99-15 & 1 & $\mathrm{~F}_{3}$ & Embrapa 1 (S) x Paraná (S) \\
\hline & JAB 99-16 & 1 & $\mathrm{~F}_{3}$ & Paraná (S) x Embrapa 1 (S) \\
\hline & JAB 99-17 & 1 & $\mathrm{~F}_{3}$ & Hartwig (R) x Paraná (S) \\
\hline & JAB 99-18 & 1 & $\mathrm{~F}_{3}$ & Embrapa 1 (S) x Hartwig (R) \\
\hline & JAB 99-19 & 1 & $\mathrm{~F}_{3}$ & Paraná (S) x Hartwig (R) \\
\hline & JAB 99-20 & 1 & $\mathrm{~F}_{3}$ & IAS-5 (S) x Paraná (S) \\
\hline & JAB 99-21 & 1 & $\mathrm{~F}_{3}$ & Hartwig (R) x Embrapa 1 (S) \\
\hline & JAB 99-40 & 1 & $\mathrm{~F}_{2}$ & FT-Cristalina (S) x BR 92-15440 (R) \\
\hline & JAB 99-41 & 1 & $\mathrm{~F}_{2}$ & BR 90-4617 (R) x FT-Cristalina (S) \\
\hline & JAB 99-42 & 1 & $\mathrm{~F}_{2}$ & BR 91-10569 (R) x FT-Cristalina (S) \\
\hline & JAB 99-43 & 1 & $\mathrm{~F}_{2}$ & Hartwig (R) x Paraná (S) \\
\hline & JAB 99-44 & 1 & $\mathrm{~F}_{2}$ & Delsoy (R) x FT-Cristalina (S) \\
\hline & JAB 99-45 & 1 & $\mathrm{~F}_{2}$ & [BR 90-4722 (R) x FT-Cristalina (S)] x FT-Cristalina (S) \\
\hline \multirow[t]{16}{*}{$2000 / 2001$} & JAB 99-10 & 5 & $\mathrm{~F}_{4}$ & FT-Cristalina (S) x BR 92-15440 (R) \\
\hline & JAB 99-11 & 3 & $\mathrm{~F}_{4}$ & BR 91-10569 (R) x FT-Cristalina (S) \\
\hline & JAB 99-12 & 4 & $\mathrm{~F}_{4}$ & BR 92-15440 (R) x FT-Cristalina (S) \\
\hline & JAB 99-13 & 3 & $\mathrm{~F}_{4}$ & Delsoy (R) x FT-Cristalina (S) \\
\hline & JAB 99-14 & 5 & $\mathrm{~F}_{4}$ & BR 90-4617 (R) x FT-Cristalina (S) \\
\hline & JAB 99-16 & 3 & $\mathrm{~F}_{4}$ & Paraná (S) x Embrapa 1 (S) \\
\hline & JAB 99-17 & 4 & $\mathrm{~F}_{4}$ & Hartwig (R) x Paraná (S) \\
\hline & JAB 99-18 & 3 & $\mathrm{~F}_{4}$ & Embrapa 1 (S) x Hartwig (R) \\
\hline & JAB 99-19 & 2 & $\mathrm{~F}_{4}$ & Paraná (S) x Hartwig (R) \\
\hline & JAB 99-20 & 5 & $\mathrm{~F}_{4}$ & IAS-5 (S) x Paraná (S) \\
\hline & JAB 99-21 & 4 & $\mathrm{~F}_{4}$ & Hartwig (R) x Embrapa 1 (S) \\
\hline & JAB 99-40 & 10 & $\mathrm{~F}_{3}$ & FT-Cristalina (S) x BR 92-15440 (R) \\
\hline & JAB 99-41 & 8 & $\mathrm{~F}_{3}$ & BR 90-4617 (R) x FT-Cristalina (S) \\
\hline & JAB 99-42 & 8 & $\mathrm{~F}_{3}$ & BR 91-10569 (R) x FT-Cristalina (S) \\
\hline & JAB 99-43 & 6 & $\mathrm{~F}_{3}$ & Hartwig (R) x Paraná (S) \\
\hline & JAB 99-44 & 10 & $\mathrm{~F}_{3}$ & Delsoy (R) x FT-Cristalina (S) \\
\hline \multirow[t]{12}{*}{$2001 / 2002$} & JAB 99-10 & 35 & $\mathrm{~F}_{5}$ & FT-Cristalina (S) x BR 92-15440 (R) \\
\hline & JAB 99-12 & 1 & $\mathrm{~F}_{5}$ & BR 92-15440 (R) x FT-Cristalina (S) \\
\hline & JAB 99-13 & 2 & $\mathrm{~F}_{5}$ & Delsoy (R) x FT-Cristalina (S) \\
\hline & JAB 99-14 & 11 & $\mathrm{~F}_{5}$ & BR 90-4617 (R) x FT-Cristalina (S) \\
\hline & JAB 99-16 & 6 & $\mathrm{~F}_{5}$ & Paraná (S) x Embrapa 1 (S) \\
\hline & JAB 99-17 & 11 & $\mathrm{~F}_{5}$ & Hartwig (R) x Paraná (S) \\
\hline & JAB 99-20 & 1 & $\mathrm{~F}_{5}$ & IAS-5 (S) x Paraná (S) \\
\hline & JAB 99-40 & 28 & $\mathrm{~F}_{4}$ & FT-Cristalina (S) x BR 92-15440 (R) \\
\hline & JAB 99-41 & 3 & $\mathrm{~F}_{4}$ & BR 90-4617 (R) x FT-Cristalina (S) \\
\hline & JAB 99-42 & 5 & $\mathrm{~F}_{4}$ & BR 91-10569 (R) x FT-Cristalina (S) \\
\hline & JAB 99-43 & 2 & $\mathrm{~F}_{4}$ & Hartwig (R) x Paraná (S) \\
\hline & JAB 99-44 & 5 & $\mathrm{~F}_{4}$ & Delsoy (R) x FT-Cristalina (S) \\
\hline \multirow[t]{6}{*}{$2002 / 2003$} & JAB 99-10 & 3 & $\mathrm{~F}_{6}$ & FT-Cristalina (S) x BR 92-15440 (R) \\
\hline & JAB 99-16 & 1 & $\mathrm{~F}_{6}$ & Paraná (S) x Embrapa 1 (S) \\
\hline & JAB 99-17 & 2 & $\mathrm{~F}_{6}$ & Hartwig (R) x Paraná (S) \\
\hline & JAB 99-40 & 3 & $\mathrm{~F}_{5}$ & FT-Cristalina (S) x BR 92-15440 (R) \\
\hline & JAB 99-43 & 1 & $\mathrm{~F}_{5}$ & Hartwig (R) x Paraná (S) \\
\hline & JAB 99-44 & 1 & $\mathrm{~F}_{5}$ & Delsoy $(\mathrm{R})$ x FT-Cristalina (S) \\
\hline
\end{tabular}

${ }^{(1)}$ Reação verificada de acordo com as proposições de Hartwig (1985); R: resistente; S: suscetível. 
ser normal e independentemente distribuído com média zero e variância $\sigma^{2}$.

O efeito fixo de conjunto refere-se a dois grupos de progênies, um deles composto por cinco progênies de gerações $F_{2}, F_{3}, F_{4}$ e $F_{5}$, e o outro composto por seis progênies de gerações $F_{3}, F_{4}, F_{5}, F_{6}$, nos anos agrícolas 1999/2000, 2000/2001, 2001/2002 e 2002/2003, respectivamente.

As análises foram realizadas utilizando-se o procedimento GLM, do programa SAS (SAS Institute, 1997).

O ensaio de avaliação de resistência ao NCS tipo 3 foi conduzido em casa de vegetação não climatizada e semeado em outubro de 2003. O delineamento adotado foi o inteiramente casualizado, com cinco repetições. Vasos de argila com capacidade de $1 \mathrm{~L}$, com substrato, foram enterrados, parcialmente, em caixas de madeira com areia lavada, para propiciar menor oscilação de temperatura no interior dos vasos. O substrato, tratado previamente com brometo de metila, foi composto por uma parte de areia fina lavada e duas partes de solo coletado na área experimental. O inóculo foi obtido de amostras de solo coletado em área naturalmente infestada pelo NCS tipo 3, localizada no Município de Iraí de Minas, MG.

Os cistos foram extraídos de amostras de solos, utilizando-se metodologia proposta por Goodey (1963). As suspensões obtidas foram lavadas, seqüencialmente, em peneiras de malha de 200 mesh e 500 mesh $(0,074 \mathrm{~mm}$ e $0,025 \mathrm{~mm})$, respectivamente. Os cistos retidos na peneira de 200 mesh foram esmagados na própria peneira por fricção, utilizando-se a extremidade oval de um tubo de ensaio.

Os ovos foram liberados dos cistos e retidos na peneira de 500 mesh. Em seguida, foram recolhidos em suspensão aquosa, em um béquer de $100 \mathrm{~mL}$, e foi retirada uma alíquota de $1 \mathrm{~mL}$ para determinação da concentração de ovos, com auxílio de uma câmara de Peters. Após as contagens, foi ajustada a concentração para 400 ovos $\mathrm{mL}^{-1}$, tendo-se realizado, então, o processo de inoculação.

Foram avaliados 15 tratamentos, com duas cultivares resistentes (Hartwig e BRSMT Caxara) e duas suscetíveis MG/BR-46 (Conquista) e FT-Cristalina, além de 11 progênies, anteriormente selecionadas, quanto aos atributos agronômicos. Aproximadamente quatro dias após a emergência, foram colocados $10 \mathrm{~mL}$ da suspensão de ovos da população do nematóide de cisto, em um orifício aberto, ao lado do colo de cada planta, com auxílio de uma pipeta automática.
Cinco plantas de cada progênie foram submetidas à inoculação, mantidas em casa de vegetação, com acompanhamento diário, em que as médias das temperaturas mínimas e das máximas diárias foram respectivamente, $22^{\circ} \mathrm{C}$ e $33^{\circ} \mathrm{C}$, por um período aproximado de 30 dias, quando foi realizada a avaliação de resistência ao nematóide de cisto da soja. Para tal, foram calculados os índices de fêmeas (IF), segundo metodologia proposta por Golden et al. (1970), em que as progênies com IF menor que $10 \%$ foram consideradas como resistentes, e aquelas com IF igual ou maior do que $10 \%$, suscetíveis. A cultivar MG/BR-46 (Conquista) foi utilizada como padrão de suscetibilidade.

\section{Resultados e Discussão}

O efeito de conjuntos, da análise de variância conjunta dos anos de plantio, em relação às variáveis estudadas, apresentou diferença altamente significativa para NDF, NV, PT, PCS, NDM, APM, VA e NN. Rocha (2002) não observou diferenças significativas entre as variáveis NDM e APM (Tabela 2).

Com relação ao efeito de gerações dentro de conjuntos (G/C), detectou-se significância a 1\% para as variáveis NV, PT, NDM, Ac e NN e a 5\% para APM.

$O$ efeito de anos foi significativo a $1 \%$, para as variáveis NV, PT, PCS, APM, VA e NN. Resultado semelhante foi observado por Rocha (2002), para a variável APM, ao estudar linhagens semiprecoces e intermediárias.

Entre as progênies, diferenças significativas a 1\% foram detectadas para NDF, NV, PT, PCS, NDM, APM, Ac, VA e NN, o que concorda, parcialmente, com os resultados obtidos por Unêda-Trevisoli (1999), que encontrou significância para NDM, APM, Ac e VA entre as progênies precoces. Resultado similar ocorreu entre as testemunhas, para todas as variáveis, exceto AIV, em que as diferenças foram significativas a $1 \%$.

Unêda-Trevisoli (1999) estudou testemunhas semiprecoces (ambiente Esalq, ano 1996/1997) e também observou diferenças altamente significativas para NDM, APM, Ac e VA.

O efeito do contraste progênies x testemunhas $(\mathrm{P}$ x T) foi não significativo apenas para Ac e NN, o que indica que, para essas variáveis, as progênies comportaramse semelhantemente às testemunhas.

A interação progênies $\mathrm{x}$ anos ( $\mathrm{P}$ x A) foi significativa a $1 \%$ para NV, PT, NDM, VA e NN, e a $5 \%$ para PCS. 
A interação (progênies $x$ testemunhas) $x$ anos [(P x T) x A], foi significativa a 1\% para NDF, NV, PT, PCS, APM, VA e NN, o que é um indicativo de que as progênies e testemunhas, em relação a essas variáveis, foram influenciadas pelas condições diferenciais dos anos, e de que o comportamento de tais genótipos é instável.

Os coeficientes de variação (CV) estimados na análise conjunta entre os anos, em ordem crescente segundo as variáveis estudadas, foram: 1,30\% (NDF), 2,22\% (NDM), 4,27\% (VA), 6,13\% (Ac), 6,30\% (NN), 7,34\% (APM), 8,22\% (NV), 9,17\% (PT), 9,59\% (PCS) е $36,23 \%$ (AIV). Os resultados relativos a NDM, APM, Ac e VA são semelhantes aos valores obtidos por UnêdaTrevisoli (1999), Yokomizo (1999) e Rocha (2002). Em relação à variável AIV, o valor observado está de acordo com Unêda-Trevisoli et al. (2002). Para NV, PT e NN, Reis et al. (2002) e Bárbaro et al. (2004) encontraram valores maiores de CV. Os resultados de CV, relativos ao NDF e PCS, são próximos aos obtidos por
Mauro et al. (1999), que encontraram valor superior para NDF e semelhante para PCS.

A Tabela 2 apresenta também os resultados das médias estimadas dos caracteres agronômicos, referentes às progênies e testemunhas comuns nos quatro anos de avaliação.

Em relação ao NDF, as menores médias entre os genótipos avaliados referiram-se à cultivar Renascença e às progênies 5 e 2 e diferiram, significativamente, das demais. Reis et al. (2002) encontraram maiores médias de NDF, com variação de valores de 60,25 a 62,26. Para $\mathrm{NV}$, observou-se que as progênies com maiores médias foram as de número 9 e 7 , apesar de não diferirem, significativamente, das demais, exceto das progênies de números 2 e 4 . Esses valores observados são discordantes dos resultados de outros trabalhos (Reis et al., 2002; Unêda-Trevisoli et al., 2002).

Para o caráter peso total por planta (PT), destacouse novamente a progênie de número 9, que apresentou a maior média para essa variável que não diferiu, signi-

Tabela 2. Quadrados médios obtidos na análise de variância conjunta, média geral, coeficiente de variação (CV), médias dos cruzamentos e testemunhas, média total dos cruzamentos $(\mathrm{Mp})$ e das testemunhas (Mt), referentes às variáveis número de dias para florescimento (NDF), número de vagens (NV), peso total por planta (PT), peso de 100 sementes (PCS), número de dias para maturidade (NDM), altura de planta na maturidade (APM), altura da inserção da primeira vagem (AIV), acamamento (Ac), valor agronômico (VA) e número de nós (NN), em cultivo de soja semeada em 9/11/1999, 23/11/2000, 22/11/2001 e 22/11/2002.

\begin{tabular}{|c|c|c|c|c|c|c|c|c|c|c|c|}
\hline \multirow[t]{2}{*}{ Fontes de variação } & \multirow[t]{2}{*}{ GL } & \multicolumn{10}{|c|}{ Quadrados médios } \\
\hline & & NDF (dias) & $\mathrm{NV}\left(\mathrm{n}^{\underline{0}}\right)$ & PT $\left(\right.$ g planta $\left.{ }^{-1}\right)$ & PCS (g) & NDM (dias) & $\mathrm{APM}(\mathrm{cm}) \quad A$ & AIV $(\mathrm{cm})$ & $\mathrm{Ac}^{(1)}$ (nota) & $\mathrm{VA}^{(1)}$ (nota) & $\mathrm{NN}\left(\mathrm{n}^{\circ}\right)$ \\
\hline Conjuntos (C) & 1 & $9,67 * *$ & $23108,73 * *$ & $206,79 * *$ & $118,22 * *$ & $730,55^{* *}$ & $1015,88^{* *}$ & $11,28^{\text {ns }}$ & $0,01^{\mathrm{ns}}$ & $0,05 * *$ & $31,84 * *$ \\
\hline Gerações $(\mathrm{G}) / \mathrm{C}$ & 3 & $0,10^{\mathrm{ns}}$ & $14352,37^{* *}$ & $885,01 * *$ & $2,49^{\mathrm{ns}}$ & $51,68 * *$ & $162,51^{*}$ & $12,26^{\mathrm{ns}}$ & $0,07 * *$ & $0,01^{\mathrm{ns}}$ & $9,79 * *$ \\
\hline $\operatorname{Anos}(\mathrm{A})$ & 3 & $1,66^{*}$ & $28818,16^{* *}$ & $3791,37 * *$ & $31,46^{* *}$ & $21,58^{\mathrm{ns}}$ & $388,30 * *$ & $42,28^{\text {ns }}$ & $0,03 *$ & $0,35 * *$ & $6,97 * *$ \\
\hline Progênies (P) & 9 & $13,17 * *$ & $3164,80 * *$ & $478,70^{* *}$ & $9,18^{* *}$ & $149,79 * *$ & $355,87 * *$ & $22,51^{\mathrm{ns}}$ & $0,05 * *$ & $0,06 * *$ & $11,13^{* *}$ \\
\hline Testemunhas (T) & 2 & $247,69 * *$ & $1726,33 * *$ & $416,70^{* *}$ & $308,06^{* *}$ & $1133,69^{* *}$ & $1327,00^{* *}$ & $456,08^{* *}$ & $0,06^{* *}$ & $0,18^{* *}$ & $72,58^{* *}$ \\
\hline $\mathrm{P} \times \mathrm{T}$ & 1 & $38,33^{* *}$ & $84417,23 * *$ & $8390,20 * *$ & $14,99 * *$ & $1339,40 * *$ & $1861,82^{* *}$ & $422,84 * *$ & $0,01^{\mathrm{ns}}$ & $0,52 * *$ & $0,09^{\mathrm{ns}}$ \\
\hline $\mathrm{P} \times \mathrm{A}$ & 27 & $0,58^{\mathrm{ns}}$ & $2037,69 * *$ & $308,14^{* *}$ & $3,34 *$ & $38,93 * *$ & $70,04^{\mathrm{ns}}$ & $8,62^{\text {ns }}$ & $0,02^{\mathrm{ns}}$ & $0,03 * *$ & $2,82 * *$ \\
\hline$(\mathrm{P} \times \mathrm{T}) \times \mathrm{A}$ & 3 & $3,13 * *$ & $20012,73^{* *}$ & $2724,96 * *$ & $13,56^{* *}$ & $5,97^{\mathrm{ns}}$ & $260,85 * *$ & $3,64^{\mathrm{ns}}$ & $0,02^{\mathrm{ns}}$ & $0,21 * *$ & $5,84 * *$ \\
\hline Resíduo & 30 & 0,40 & 70,53 & 6,39 & 1,61 & 8,52 & 40,38 & 39,62 & 0,01 & 0,01 & 0,96 \\
\hline Média geral & & 48,51 & 102,16 & 27,57 & 13,23 & 131,34 & 86,50 & 17,38 & 1,49 & 1,81 & 15,54 \\
\hline CV $(\%)$ & & 1,30 & 8,22 & 9,17 & 9,59 & 2,22 & 7,34 & 36,23 & 6,13 & 4,27 & 6,30 \\
\hline Progênie & Número & \multicolumn{10}{|c|}{ Médias $^{(2)}$} \\
\hline JAB 99-10-1-2-5 & 1 & $46,75 \mathrm{ef}$ & $105,25 \mathrm{abc}$ & $28,12 \mathrm{abc}$ & $14,05 \mathrm{bcd}$ & $126,25 b c$ & $87,25 \mathrm{abcd}$ & $14,25 \mathrm{ab}$ & $1,57 \mathrm{ab}$ & $1,70 \mathrm{~b}$ & $16,00 \mathrm{abcd}$ \\
\hline JAB 99-10-2-4-2 & 2 & $45,75 \mathrm{fgh}$ & $102,50 \mathrm{bc}$ & $27,90 \mathrm{abc}$ & $14,83 \mathrm{abcd}$ & $125,75 b c$ & $87,50 \mathrm{abcd}$ & $13,50 \mathrm{~b}$ & $1,62 \mathrm{a}$ & $1,67 \mathrm{~b}$ & $14,00 \mathrm{~cd}$ \\
\hline JAB 99-10-3-8-2 & 3 & $48,75 \mathrm{~cd}$ & $117,75 \mathrm{abc}$ & $31,81 \mathrm{abc}$ & $14,62 \mathrm{abcd}$ & $126,25 \mathrm{bc}$ & $83,00 \mathrm{abcde}$ & $15,25 \mathrm{ab}$ & $1,62 \mathrm{a}$ & $1,77 \mathrm{ab}$ & $15,75 \mathrm{abcd}$ \\
\hline JAB 99-16-4-23-18 & 4 & $50,00 \mathrm{bc}$ & $88,50 \mathrm{bc}$ & $38,70 \mathrm{abc}$ & $18,04 \mathrm{a}$ & $120,50 \mathrm{bc}$ & $70,75 \mathrm{de}$ & $20,00 \mathrm{ab}$ & $1,37 \mathrm{abc}$ & $1,95 \mathrm{ab}$ & $15,25 \mathrm{bcd}$ \\
\hline JAB 99-17-3-3-5 & 5 & $44,75 \mathrm{gh}$ & $115,25 \mathrm{abc}$ & $35,02 \mathrm{abc}$ & $14,81 \mathrm{abcd}$ & $118,50 \mathrm{c}$ & $66,50 \mathrm{e}$ & $14,75 \mathrm{ab}$ & $1,30 \mathrm{c}$ & $1,92 \mathrm{ab}$ & $13,00 \mathrm{~d}$ \\
\hline JAB 99-17-4-9-1 & 6 & $48,75 \mathrm{~cd}$ & $134,50 \mathrm{abc}$ & $47,58 \mathrm{ab}$ & $14,36 \mathrm{bcd}$ & $126,25 \mathrm{bc}$ & $71,50 \mathrm{de}$ & $12,25 b$ & $1,35 b c$ & $2,07 \mathrm{a}$ & $14,75 \mathrm{bcd}$ \\
\hline JAB 99-40-3-32-3 & 7 & $46,25 \mathrm{fg}$ & $196,00 \mathrm{a}$ & $32,85 \mathrm{abc}$ & $9,40 \mathrm{ef}$ & $126,50 \mathrm{bc}$ & $96,25 \mathrm{ab}$ & $17,75 \mathrm{ab}$ & $1,47 \mathrm{abc}$ & $1,92 \mathrm{ab}$ & $17,25 \mathrm{abc}$ \\
\hline JAB 99-40-5-17-20 & 8 & $48,50 \mathrm{~cd}$ & $129,75 \mathrm{abc}$ & $29,92 \mathrm{abc}$ & $12,43 \mathrm{cde}$ & $126,50 \mathrm{bc}$ & 83,50 abcde & $14,25 \mathrm{ab}$ & $1,57 \mathrm{ab}$ & $1,97 \mathrm{ab}$ & $13,25 \mathrm{~d}$ \\
\hline JAB 99-40-12-1-2 & 9 & $48,25 \mathrm{de}$ & $195,00 \mathrm{a}$ & $62,23 \mathrm{a}$ & $12,31 \mathrm{cde}$ & $131,25 \mathrm{~b}$ & $98,75 \mathrm{ab}$ & $16,75 \mathrm{ab}$ & $1,50 \mathrm{abc}$ & $1,80 \mathrm{ab}$ & $19,00 \mathrm{a}$ \\
\hline JAB 99-43-11-15-20 & 10 & $48,50 \mathrm{~cd}$ & $110,50 \mathrm{abc}$ & $27,98 \mathrm{abc}$ & $11,36 \mathrm{de}$ & $129,75 b$ & $76,25 \mathrm{cde}$ & $14,50 \mathrm{ab}$ & $1,45 \mathrm{abc}$ & $1,95 \mathrm{ab}$ & $14,50 \mathrm{bcd}$ \\
\hline JAB 99-44-12-23-1 & 11 & $50,50 \mathrm{~b}$ & $152,00 \mathrm{ab}$ & $43,06 a b c$ & $13,64 \mathrm{bcd}$ & $146,50 \mathrm{a}$ & 82,25 bcde & $16,00 \mathrm{ab}$ & $1,55 \mathrm{abc}$ & $1,95 \mathrm{ab}$ & $17,75 \mathrm{ab}$ \\
\hline $\mathrm{Mp}$ & & 47,89 & 131,56 & 36,83 & 13,63 & 127,64 & 82,13 & 15,39 & 1,49 & 1,88 & 15,51 \\
\hline Liderança & & $50,00 \mathrm{bc}$ & $59,92 \mathrm{bc}$ & $16,95 \mathrm{bc}$ & $15,01 \mathrm{abc}$ & $130,58 b$ & $92,50 \mathrm{abc}$ & $21,33 \mathrm{ab}$ & $1,40 \mathrm{abc}$ & $1,70 b$ & $15,41 \mathrm{abcd}$ \\
\hline FT-Cristalina & & $53,42 a$ & $80,08 b c$ & $10,03 \mathrm{c}$ & $6,95 \mathrm{f}$ & $148,00 \mathrm{a}$ & $102,00 \mathrm{a}$ & $25,25 \mathrm{a}$ & 1,51abc & $1,60 \mathrm{~b}$ & $18,00 \mathrm{ab}$ \\
\hline Renascença & & $44,41 \mathrm{~h}$ & $58,75 \mathrm{c}$ & $21,75 \mathrm{bc}$ & $16,29 \mathrm{ab}$ & $129,00 \mathrm{bc}$ & $81,00 \mathrm{bcde}$ & $13,16 \mathrm{~b}$ & $1,54 \mathrm{abc}$ & $1,85 \mathrm{ab}$ & $13,08 \mathrm{~d}$ \\
\hline Mt & & 49,27 & 66,24 & 16,25 & 12,76 & 135,86 & 91,83 & 19,92 & 1,48 & 1,72 & 15,50 \\
\hline
\end{tabular}

(1)Análise realizada com dados transformados para $\sqrt{x}$, com $\mathrm{x}=1$ a 5 (Ac e VA). (2)Médias, de 4 ambientes e 5 repetições, seguidas de mesma letra,

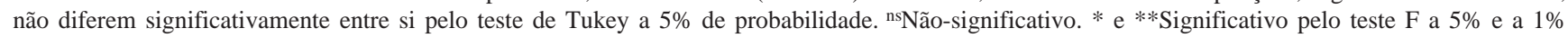
de probabilidade, respectivamente. 
ficativamente, das médias das demais progênies. Em contraposição, Unêda-Trevisoli et al. (2002) encontraram valores bem inferiores, com variação de 3,55 a 4,71 .

Quanto ao peso de 100 sementes (PCS), observouse que a progênie de número 4 apresentou valor médio significativamente superior $(\mathrm{P}<0,01)$ aos apresentados pelas demais progênies, com exceção das progênies de números 2, 3 e 5 .

O menor número de dias para maturidade (NDM) foi detectado na progênie de número 5 , que apresentou menor média entre todos os genótipos avaliados, o que seria um indicativo de precocidade. Dados concordantes com relação ao NDM foram encontrados por Reis et al. (2002).

Para a variável altura de planta na maturidade (APM), foram observadas diferenças significativas $(P<0,01)$ entre as progênies, porém todas apresentaram médias de APM acima de $60 \mathrm{~cm}$, com estimativas entre 66,50 cm (progênie de número 5), e 98,75cm (progênie de número 9), as quais podem ser consideradas desejáveis para colheita mecanizada, segundo Bonetti (1983). Rocha (2002) ao estudar linhagens precoces, semiprecoces, intermediárias e semitardias relatou valores superiores.

Quanto à variável altura da inserção da primeira vagem (AIV), todas as progênies apresentaram médias iguais ou maiores do que $12 \mathrm{~cm}$, que é o recomendável para a cultura (São Paulo, 1990).

Em relação à variável Ac, destacou-se a progênie de número 5 , que apresentou média menor do que os demais genótipos, próxima ao valor encontrado por Costa et al. (2004).
Quanto ao valor agronômico (VA), a progênie de número 6 apresentou maior média, com estimativa acima da média geral das progênies (Mp) e das testemunhas (Mt). Tais valores são similares aos relatados por UnêdaTrevisoli (1999). Mesmo tendo havido variação entre todos os genótipos avaliados, o número de nós mostrouse mais alto para a progênie de número 9. Reis et al. (2002) encontraram valor semelhante aos obtidos nesta análise $(13,23)$.

As progênies de números 1, 2, 3, 5, 8 e 9, revelaramse resistentes ao tipo 3 do NCS, por apresentarem índices de fêmeas menores do que $10 \%$, conforme proposto por Golden et al. (1970) (Tabela 3). Esse resultado pode ser atribuído ao fato de que essas progênies são resultantes de cruzamentos, nos quais um dos parentais apresenta resistência ao patógeno. As progênies de números 11, 10 e 4, por apresentarem índices de fêmeas com variação entre 15,7\% e 24,2\%, e as progênies 7 e 6 , com índices acima de $25 \%$, foram classificadas como moderadamente resistentes e suscetíveis, respectivamente, segundo a modificação efetuada por Yamashita et al. (1999) na proposta de Golden et al. (1970). Entretanto, deve-se tomar por base a proposta mais rigorosa que, no caso, seria a de Golden et al. (1970), por tratar de um nematóide agressivo. A indicação de progênies classificadas como moderadamente resistentes, de acordo com Yamashita et al. (1999), em programas de melhoramento visando resistência à referida doença, não é aconselhável.

Resultados similares foram obtidos por Arantes et al. (1998), Campos et al. (2003) e Mauro et al. (2004) em trabalhos realizados com soja, utilizando-se da mesma técnica de avaliação do patógeno e objetivo semelhante, tendo confirmada sua eficiência.

Tabela 3. Número de fêmeas por planta, índice de fêmeas (IF) e reação das testemunhas e das progênies de soja com inoculação do nematóide de cisto da soja (tipo 3), em cultivo semeado em 17/10/2003, em casa de vegetação não climatizada(1).

\begin{tabular}{|c|c|c|c|c|c|c|c|c|c|c|}
\hline \multirow[t]{2}{*}{ Progênie/Testemunha } & \multirow[t]{2}{*}{ Número } & \multirow[t]{2}{*}{ Geração } & \multicolumn{5}{|c|}{ Número de fêmeas/repetição } & \multirow[t]{2}{*}{ Média } & \multirow[t]{2}{*}{ IF $(\%)$} & \multirow[t]{2}{*}{ Reação } \\
\hline & & & I & II & III & IV & $\mathrm{V}$ & & & \\
\hline JAB 99-10-1-2-5 & 1 & $\mathrm{~F}_{7}$ & 0,0 & 0,0 & 0,0 & 0,0 & 0,0 & 0,0 & 0,0 & $\mathrm{R}$ \\
\hline JAB 99-10-2-4-2 & 2 & $\mathrm{~F}_{7}$ & 0,0 & 0,0 & 0,0 & 0,0 & 0,0 & 0,0 & 0,0 & $\mathrm{R}$ \\
\hline JAB 99-10-3-8-2 & 3 & $\mathrm{~F}_{7}$ & 0,0 & 0,0 & 0,0 & 0,0 & 0,0 & 0,0 & 0,0 & $\mathrm{R}$ \\
\hline JAB 99-16-4-23-18 & 4 & $\mathrm{~F}_{7}$ & 37,0 & 26,0 & 13,0 & 9,0 & 52,0 & 27,4 & 24,2 & $\mathrm{~S}$ \\
\hline JAB 99-17-3-3-5 & 5 & $\mathrm{~F}_{7}$ & 9,0 & 14,0 & 5,0 & 11,0 & 8,0 & 9,4 & 8,2 & $\mathrm{R}$ \\
\hline JAB 99-17-4-9-1 & 6 & $\mathrm{~F}_{7}$ & 47,0 & 36,0 & 75,0 & 13,0 & 61,0 & 46,4 & 40,9 & $\mathrm{~S}$ \\
\hline JAB 99-40-3-32-3 & 7 & $\mathrm{~F}_{6}$ & 53,0 & 65,0 & 44,0 & 32,0 & 27,0 & 44,2 & 38,9 & $\mathrm{~S}$ \\
\hline JAB 99-40-5-17-20 & 8 & $\mathrm{~F}_{6}$ & 12,0 & 15,0 & 3,0 & 7,0 & 4,0 & 8,2 & 7,2 & $\mathrm{R}$ \\
\hline JAB 99-40-12-1-2 & 9 & $\mathrm{~F}_{6}$ & 0,0 & 7,0 & 2,0 & 0,0 & 2,0 & 2,2 & 1,9 & $\mathrm{R}$ \\
\hline JAB 99-43-11-15-20 & 10 & $\mathrm{~F}_{6}$ & 11,0 & 8,0 & 15,0 & 9,0 & 66,0 & 21,8 & 19,2 & $\mathrm{~S}$ \\
\hline JAB 99-44-12-23-1 & 11 & $\mathrm{~F}_{6}$ & 3,0 & 2,0 & 46,0 & 7,0 & 31,0 & 17,8 & 15,7 & $\mathrm{~S}$ \\
\hline BRSMT Caxara & & & 0,0 & 0,0 & 0,0 & 0,0 & 0,0 & 0,0 & 0,0 & $\mathrm{R}$ \\
\hline Hartwig & & & 0,0 & 0,0 & 0,0 & 0,0 & 0,0 & 0,0 & 0,0 & $\mathrm{R}$ \\
\hline MG/BR-46 Conquista (P) & & & 107,0 & 91,0 & 139,0 & 126,0 & 104,0 & 113,4 & 100,0 & $\mathrm{~S}$ \\
\hline FT-Cristalina & & & 108,0 & 137,0 & 125,0 & 142,0 & 94,0 & 121,2 & 106,9 & $\mathrm{~S}$ \\
\hline
\end{tabular}

(1)IF (\%) = (Número médio de fêmeas na progênie/Número médio de fêmeas na cultivar Conquista) x 100; R (resistente): IF (\%) <10\%; S (suscetível): IF (\%) $\geq 10 \%$; (P) Padrão de suscetibilidade. 


\section{Conclusões}

1. As progênies de soja mais promissoras, quanto aos atributos agronômicos, são JAB 99-17-4-9-1 e JAB 9940-12-1-2.

2. A progênie JAB 99-40-12-1-2 demonstra potencial para ser utilizada em fase subseqüente do programa de melhoramento, visando-se resistência ao NCS (tipo 3).

3. A técnica utilizada é eficiente na discriminação de progênies suscetíveis e resistentes ao NCS (tipo 3).

\section{Referências}

ARANTES, N.E.; KIIHL, R.A.S.; ALMEIDA, L.A. Melhoramento genético visando à resistência. In: SOCIEDADE BRASILEIRA DE NEMATOLOGIA (Ed.). O nematóide de cisto da soja: a experiência brasileira. Jaboticabal: Artsingner, 1999. p.105-117.

ARANTES, N.E.; MAURO, A.O.; TIHOHOOD, D. An alternative field method for screening soybean genotypes for resistance to Heterodera glycines. Journal of Nematology, v.30, p.542-546, 1998.

BÁRBARO, I.M.; MAURO, A.O.; CENTURION, M.A.P.C.; PERECIN, D.; UNÊDA-TREVISOLI, S.H.; MUNIZ, F.R.S.; COSTA, M.M.; GAVIOLI, E.A. Seleção em populações $F_{3}$ de soja para resistência ao cancro da haste e bons atributos agronômicos. Revista Ceres, v.51, p.619-634, 2004.

BONETTI, L.P. Cultivares e seu melhoramento genético. In: VERNETTI, F.J. (Coord.). Soja, genética e melhoramento. Campinas: Fundação Cargill, 1983. v.2, p.741-800.

CAMPOS, F.L.; DI MAURO, A.O.; CENTURION, M.A.P.C.; UNÊDA-TREVISOLI, S.H.; OLIVEIRA, R.C. Adaptabilidade, estabilidade e resistência ao cancro da haste em soja. Científica, v.32, p.171-177, 2003.

CONAB. Comparativo da área, produção e produtividade. Disponível em: <http://www.conab.gov.br>. Acesso em: 10 jan. 2004.

COSTA, M.M.; DI MAURO, A.O.; UNÊDA-TREVISOLI, S.H.; ARRIEL, N.H.C.; BÁRBARO, I.M.; MUNIZ, F.R.S. Ganho genético por diferentes critérios de seleção em populações segregantes de soja. Pesquisa Agropecuária Brasileira, v.39, p.1095-1102, 2004.

DIAS, W.P. Genética da resistência da soja à raça $\mathbf{4}^{+}$do nematóide de cisto, Heterodera glycines. 2003. 83p. Dissertação (Doutorado) - Universidade Federal de Lavras, Lavras.

EMBRAPA. Centro Nacional de Pesquisa de Soja. Recomendações técnicas para a cultura da soja na Região Central do Brasil 1998/99. Londrina: Embrapa-CNPS, 1998. 182p. (Documentos, 120). GOLDEN, A.M.; EPPS, J.M.; RIGGS, R.D.; DUCLOS, L.A.; FOX, J.A.; BERNARD, R.L. Terminology and identity of infraspecific forms of the soybean cyst nematode (Heterodera glycines). Plant Disease Reporter, v.54, p.544-546, 1970.

GOODEY, J.B. Estimating Heterodera populations in the soil. In: GOODEY, J.B. Laboratory methods for work with plant and soil nematodes. London: Her Majesty’s Stationery Office, 1963. p.1256. (Technical bulletin, 2).

MAURO, A.O.; COSTA L.C.; PERECIN, D. Análises genéticas no desenvolvimento de variedades de soja para cultivo em áreas de reforma de canavial. Revista Ceres, v.46, p.423-433, 1999.

MAURO, A.O.; UNÊDA-TREVISOLI, S.H.; MAURO, S.M.Z.; COSTA, M.M.; OLIVEIRA, R.C.; ARANTES, N.E. Efficiency of microsatellite markers in assisted selection for resistance to soybean cyst nematode (race 3). Crop Breeding and Applied Biotechnology, v.4, p.28-34, 2004.

REIS, E.F. dos; REIS, M.S.; SEDIYAMA, T.; CRUZ, C.D. Estimativa de variâncias e herdabilidades de algumas características primárias e secundárias da produção de grãos em soja (Glycine max (L.) Merrill). Ciência Agrotécnica, v.26, p.749-761, 2002.

ROCHA, M.M. Seleção de linhagens experimentais de soja para adaptabilidade e estabilidade fenotípica. 2002. 173p. Tese (Doutorado) - Escola Superior de Agricultura Luiz de Queiroz, Piracicaba.

SÃO PAULO. Secretaria de Agricultura e Abastecimento. Regimento interno do sistema de avaliação e recomendação de cultivares de soja para o Estado de São Paulo. São Paulo, 1990. 15p.

SAS INSTITUTE (Cary, Estados Unidos). Sas/Stat software: changes and enhancements through release 6.12. Cary, 1997.

UNÊDA-TREVISOLI, S.H. Estabilidade fenotípica e potencialidade de progênies obtidas por cruzamentos óctuplos em soja. 1999. 228p. Tese (Doutorado) - Escola Superior de Agricultura Luiz de Queiroz, Piracicaba.

UNÊDA-TREVISOLI, S.H.; DI MAURO, A.O.; COSTA, M.M.; ARIEL, N.H.C.; CAPELOTO, A.; BÁRBARO, I.M.; MUÑIZ, F.R.S. Avaliação de herança de resistência ao oídio (Microsphaera diffusa) e do potencial agronômico em populações de soja. Revista Brasileira de Oleaginosas e Fibrosas, v.6, p.627-634, 2002.

YAMASHITA, O.M.; SILVA, J.F.V.S.; DIAS, W.P.; GOULART, A.M.C. Reação de genótipos de soja tipo alimento ao nematóide de cisto da soja, Heterodera glycines e ao nematóide de galha, Meloidogyne javanica. Nematologia Brasileira, v.23, p.17-24, 1999.

YOKOMIZO, G.K. Interação genótipos $\mathbf{x}$ ambientes em topocruzamentos de soja tipo alimento com tipo grão. 1999. 171p. Tese (Doutorado) - Escola Superior de Agricultura Luiz de Queiroz, Piracicaba.

YORINORI, J.T. Riscos de surgimento de novas doenças na cultura da soja. In: CONGRESSO DE TECNOLOGIA E COMPETITIVIDADE DA SOJA NO MERCADO GLOBAL, 2000, Cuiabá. Anais. Cuiabá: Fundação MT, 2000. p.165-169.

Recebido em 28 de abril de 2004 e aprovado em 7 de março de 2005 